\title{
Rates and Predictors of Failure of First-line Antiretroviral Therapy and Switch to Second-line ART in South Africa
}

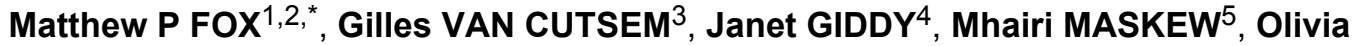 \\ KEISER $^{6}$, Hans PROZESKY ${ }^{7}$, Robin WOOD ${ }^{8}$, Miguel A. HERNÁN ${ }^{9,10}$, Jonathan A C \\ STERNE ${ }^{11}$, Matthias EGGER ${ }^{6}$, and Andrew BOULLE ${ }^{12}$ for the leDEA-SA collaboration
}

${ }^{1}$ Center for Global Health and Development, Boston University, Boston, MA, USA ${ }^{2}$ Boston University School of Public Health, Boston University, Boston, MA, USA ${ }^{3}$ Medicines Sans Frontier, Cape Town, South Africa ${ }^{4}$ McCord Hospital, Durban, South Africa ${ }^{5}$ Clinical HIV Research Unit, University of the Witwatersrand, Johannesburg, South Africa ${ }^{6}$ Institute of Social and Preventive Medicine, University of Bern, Bern, Switzerland ${ }^{7}$ Department of Medicine, University of Stellenbosch and Tygerberg Academic Hospital, Cape Town, South Africa ${ }^{8}$ Desmond Tutu HIV Centre, University of Cape Town, Cape Town, South Africa ${ }^{9}$ Department of Epidemiology, Harvard School of Public Health, Boston, MA ${ }^{10}$ Harvard-MIT Division of Health Sciences and Technology, Boston, MA ${ }^{11}$ School of Social and Community Medicine, University of Bristol, Bristol, UK ${ }^{12}$ Centre for Infectious Disease Epidemiology and Research, School of Public Health and Family Medicine, University of Cape Town, Cape Town, South Africa

\section{Abstract \\ Objectives-To measure rates and predictors of virologic failure and switch to second-line ART in South Africa.

\begin{abstract}
Design-Observational cohort study
Methods-We included ART-naïve adult patients initiated on public-sector ART (Jan 2000-July 2008) at five sites in South Africa who completed $\geq 6$ months of follow-up. We estimated cumulative risk of virologic failure (viral load $\geq 400$ copies $/ \mathrm{ml}$ with confirmation above varying thresholds) and switching to second-line ART.
\end{abstract}

Results-19,645 patients (29,935 person-years) had a median of 1.3 years of study follow-up (1.8 years on ART) and a median CD4 count of 96 (IQR:40-159) cells/ $\mu 1$ at ART initiation. 9.9\% (4.5/100 person-years) failed ART in median 16 (IQR:12-23) months since ART initiation, with median 2.9 (IQR:1.8-5.0) months between first elevated and confirmatory viral loads. By survival analysis, using a confirmatory threshold of 400 copies $/ \mathrm{ml}, 16.9 \%$ (95\%CI:15.4-18.6\%) failed by

\footnotetext{
*Author for correspondence: Crosstown Center, $3^{\text {rd }}$ Floor, 801 Massachusetts Ave, Boston, MA 02118, USA, mfox@bu.edu, $+1-617-414-1270$.

Conflicts of Interest

All authors state that they have no conflict of interest.

IeDEA Southern Africa Steering Group and site principal investigators:

Cape Town, South Africa: Brian Eley, Red Cross Children's Hospital; Daniele Garone, Khayelitsha ART Programme and Médecins Sans Frontières; Robin Wood, Gugulethu and Masiphumelele ART Programmes and Desmond Tutu HIV Centre; Hans Prozesky, Tygerberg Academic Hospital. Johannesburg, South Africa: Christopher Hoffmann, Aurum Institute for Health Research; Patrick MacPhail, Themba Lethu Clinic, Helen Joseph Hospital; Harry Moultrie, Wits Reproductive Health and HIV Institute; Karl Technau, Rahima Moosa Mother and Child Hospital. KwaZulu-Natal, South Africa: James Ndirangu, Hlabisa HIV Treatment and Care Programme; Janet Giddy, McCord Hospital, Durban. Zimbabwe: Cleophas Chimbetete, Newlands Clinic, Harare; Christiane Fritz, SolidarMed Zimbabwe. Malawi: Sam Phiri, Lighthouse Clinic, Lilongwe. Mozambique: Sabrina Pestilli, SolidarMed Mozambique; Paula Vaz, Paediatric Day Hospital, Maputo. Zambia: Jeff Stringer, Center for Infectious Disease Research
} 
five years on ART, but only 7.8\% (95\%CI:6.6\%-9.3\%) using a threshold of 10,000. CD4 <25 vs. 100-199 (adjusted HR:1.57;95\%CI 1.35-1.83), ART initiation viral load $\geq 1,000,000$ vs. $<10,000$, $(1.32 ; 0.91-1.93)$ and $2+$ gaps in care vs. 0 (95\% CI:6.61; 4.52-9.68) were predictive of failure. Overall 10.1\% (95\%CI:9.0\%-11.4\%) switched to second-line by five years on ART. Lower CD4 at failure and higher rate of CD4 decline were predictive of switch (decline 100\% to $51 \%$ vs. $25 \%$ to $-25 \%$, adjusted HR:1.96;95\%CI:1.35-2.85).

Conclusions-In resource-limited settings with viral load monitoring, virologic failure rates are highly sensitive to thresholds for confirmation. Despite clear guidelines there is considerable variability in switching failing patients, partially in response to immunologic status and postfailure evolution.

\section{Keywords}

HIV; AIDS; antiretroviral therapy; viral load; virologic treatment failure; second line

\section{INTRODUCTION}

As the global scale-up of antiretroviral therapy (ART) has reached nearly five million people, ${ }^{1}$ a growing body of evidence from large observational cohorts has demonstrated positive clinical, immunologic and virologic outcomes being achieved throughout subSaharan Africa. ${ }^{2-7}$ Even though large numbers of new patients are still starting treatment in resource-limited settings, focus is shifting from the short-term stresses of treatment initiation to the long-term problems of managing a lifelong chronic disease. A critical part of this shift is an emphasis on managing the growing number of public-sector patients who already have failed or will soon fail first-line therapy. ${ }^{8-13}$

While recent estimates suggest only $2 \%$ of those currently on ART are on a second-line regimen, ${ }^{14}$ a far greater number are likely to be failing virologically but have not switched from first-line therapy: WHO estimates that 500,000 to 800,000 patients required switching to second-line regimens by $2010 .{ }^{15}$ As ART scale-up continues and the average duration on ART increases, both the absolute number and relative proportion of patients needing secondline therapy continues to grow. It has previously been estimated in South Africa that by 5years on ART $14 \%$ of patients fail virologically. ${ }^{2}$

Poor access to HIV-1 RNA viral load testing is a key challenge facing many national programs 12,16 and therefore reliance on clinical and immunologic means of determining when to switch, which have poor predictive ability for virologic failure, is problematic. ${ }^{17-22}$ There is increasing pressure to improve access to viral load testing in high burden settings, and we recently reported improved outcomes in programmes in Southern Africa which utilize routine viral load testing. ${ }^{12}$ South Africa is one of the few high-burden settings to follow the public health approach to ART service delivery, with access to routine viral load monitoring and a standardized approach to confirming virologic failure. In settings with access to viral load testing, WHO recommends switching therapy when a patient has a persistent viral load above 5,000 viral copies $/ \mathrm{ml}^{1}$. The performance of this guidance at a national level, and the programme-level impact of different thresholds for determining virologic failure have not previously been described.

Individual cohorts have reported on durability of first-line regimens, associations with confirmed virologic failure, and delays in switching to second-line, but there is limited understanding of factors predicting switch after virologic failure, and how consistent practice is with respect to switching failing patients to second-line therapy. ${ }^{12} \mathrm{We}$ examined the impact of the definition of virologic failure on failure rates, quantified rates of virologic failure and switch to second-line ART, and studied predictors of each in a combined cohort 
of nearly 20,000 patients initiating ART throughout South Africa. We also explored variability in switching rates by treatment program.

\section{METHODS}

\section{Study Design and Population}

The data for this analysis come from the IeDEA-SA collaboration (http://www.iedea-sa.org/), a collaboration of HIV treatment programmes in Southern Africa combining prospectively collected clinical treatment data from 25 programmes. ${ }^{23}$ At the time of data transfer, participating sites represented nearly $10 \%$ of all South African adult public-sector patients initiating ART. ${ }^{24}$ We included data from five programs, operating throughout the country, with adequate viral load data. All clinics followed the 2004 South African National Treatment Guidelines for public-sector ART provision. ${ }^{25}$ While these guidelines have recently been revised, ${ }^{26}$ during the study period patients whose CD4 cell count declined to below $200 \mathrm{cells} / \mathrm{ml}^{3}$ or who were diagnosed with a WHO Stage IV condition (excluding extra-pulmonary tuberculosis) were eligible for ART. Standard firstline regimens comprised stavudine (d4T) with lamivudine (3TC) and either efavirenz (EFV) or nevirapine (NVP). Zidovudine (AZT) was available for specific indications. Once on ART, guidelines provided for 6-monthly viral load and CD4 count monitoring. In four of the five sites viral load testing was done by the National Health Laboratory Service (NucliSens EasyQ HIV-1 assay; bioMérieux, Boxtel, The Netherlands), while in Gugulethu a private laboratory provided the viral load testing (Bayer HIV-1 RNA 3.0 assay, Leverkusen, Germany). Virological failure was defined as a two consecutive viral loads above 400 copies $/ \mathrm{mL}$, with the second value $>5000$ copies $/ \mathrm{mL}$ despite stepped-up adherence interventions. The recommended second-line regimen was AZT, didanosine (ddi) and lopinavir-ritonavir (LPVr). There was some variation in the application of these guidelines, largely because some sites initiated ART prior to formalization of national guidelines. This included using AZT over d4T, the timing of the first monitoring viral load, the frequency of monitoring and the sequential thresholds used to designate confirmed virologic failure (Table 1). Sites nevertheless closely followed a uniform national programme with respect to ART eligibility, patient preparation, regimens, monitoring frequency and failure definitions, ${ }^{27}$ facilitating this combined analysis.

\section{Analytic Strategy}

Eligible patients were ART naïve adults aged $\geq 16$ initiated on a standard first-line ART regimen at one of the five sites between Jan 2000 and July 2008, and who completed $\geq 6$ months of follow-up after ART initiation. We conducted three analyses: of 1) time to virologic treatment failure in which we varied the failure definition; 2 ) the rate and predictors of treatment failure using a common virologic failure definition; and 3) time to and predictors of switching to second-line ART among those failing first-line. For the timeto-switch analysis we included only patients in care for six-months after documented virologic failure. Patients who were never virologically suppressed were included in the main analyses, but were excluded in a sensitivity analysis. Viral load testing completeness at 2 years duration on ART was estimated for each cohort for all patients followed up for at least 27 months based on viral loads taken between 21 and 27 months on ART.

For analysis 1 , we defined treatment failure as a detectable viral load ( $\geq 400$ copies $/ \mathrm{ml})$ after 6 months on first-line ART followed by a second consecutive viral load above a threshold (which varied) separated by between 2 weeks and 1 year without suppression during that time. We varied the threshold for the second elevated value $(\geq 400, \geq 1000, \geq 5000, \geq 10,000$ copies $/ \mathrm{mL})$. Patients who died after a first elevated viral load $(\mathrm{N}=79)$ with no further elevated viral load were not considered failures. Because some patients who were likely 
virologic failures died or left care before receiving a confirmatory viral load, in sensitivity analyses we analyzed rates of confirmed failure or death after initial viral load elevation to account for missed failures in patients who died. For analysis 2 we used a threshold of $\geq 1000$ (referred to as the "common failure definition"), because no cohort had a protocol requiring switching below this threshold, which is now used in the South African national ART programme..$^{28}$ Analysis 3 was limited to patients meeting their clinic's failure definition (see Table 1), because sites typically would not switch patients who had not met their definition. We defined switching to second-line ART as initiating a PI with a change in at least one nucleoside reverse transcriptase inhibitor (NRTI), $\geq 6$-months after starting firstline therapy.

We defined treatment gaps as the number of days between the end date of all drugs in one prescribed regimen and the start date of the next regimen. We defined CD4 count at ART initiation as the last measure 12 months before through 14 days after initiation (87\% of patients had such a measure). We defined viral load at ART initiation as the last viral load measure 6 months before through 3 days after ART initiation. For predictors of switching, CD4 count and viral load at failure were defined as the temporally last measures 3 months before through 2 weeks after the second detectable failing viral load. Because we hypothesized clinicians would prioritize switching failing patients with low or dropping CD4 counts, we include time updated "current" CD4 count after failure and CD4 decline as predictors in multivariable models. For missing current CD4 counts, we carried forward the last measure up to 9 months.

For failure analyses, person-time accrued from six months after initiating ART until the earliest of death, loss to follow-up (LTFU), five years on ART, administrative censoring (which varied by cohort but at latest was Jan 2009), or treatment failure (date of the second elevated viral load). For switching analyses, person-time accrued from treatment failure until the earliest of death, LTFU, administrative censoring, or switch. LTFU was determined to have occurred at the last recorded visit and was defined based on each of the individual sites' definitions.

\section{Statistical Methods}

We derived Kaplan-Meier estimates of cumulative failure probabilities using the differing failure definitions and stratified by predictors of failure. We estimated hazard ratios (HR) for associations of patient characteristics with both virologic treatment failure and switch to second-line ART using Cox proportional hazards regression. In addition to age and sex, we included variables with a univariate p-value $<0.2$ in multivariable models. For failure models we included cohort, ART initiation year, NNRTI in first-line ART (EFV/NVP), TB treatment (Yes/No/Missing), WHO stage (I/II, III/IV, missing), CD4 count and viral load at ART initiation and treatment interruptions of 7 days or more. For switch models we included cohort, year of failure, years on ART at failure, current CD4 count, \% change in CD4 count from failure, and viral load at failure. As a sensitivity analysis, missing baseline values were also imputed using fully conditional modeling by means of a chained equations approach, ${ }^{29}$ with estimation results combined by Rubin's rules. ${ }^{30}$

\section{Ethics}

Approval for analyses was given by the Universities of Cape Town, Bern and Boston University. All sites had ethical approval to transfer anonymized data to the IeDEA data center. 


\section{RESULTS}

Details of the five public-sector treatment sites in South Africa are given in Table 1. While some patients initiated treatment as early as 1999, the majority initiated treatment since the public-sector ART rollout in South Africa began in April 2004. Viral load testing completeness at two years on ART ranged from $71 \%$ to $85 \%$.

\section{Cohort description}

Of 23,465 adults completing 6 months of ART, 3,820 were excluded because they initiated a nonstandard first-line regimen or their first-line regimen could not be determined. The 19,645 eligible patients were followed for 29,935 person-years (py) (median (range across sites) 1.3 years (1.1-1.4) in the study and 1.8 years on ART). Excluded patients had similar age, gender, cohort, TB treatment at ART initiation to eligible patients, but were more likely to have initiated ART before 2004 (19\% vs. 5\%), be WHO Stage I/II (47\% vs. 38\%) and have CD4 count $>350$ at ART initiation (27\% vs. $1 \%$ ).

Two programs accounted for $73 \%$ of all patients (Table 2). The majority of patients were female (66\%), initiated ART after $2005(>51 \%)$, and initiated d4T-3TC-EFV $(68 \%)$ or d4T-3TC-NVP (21\%). Advanced immunosuppression was common at ART initiation: 63\% of patients were WHO Stage III/IV, $30 \%$ had a CD4 count $<50$ and $36 \%$ were on tuberculosis treatment. Median CD4 count at ART initiation was 93 (IQR:39-155) cells/ $\mu 1$. Of 19,456 patients with known outcome $79 \%$ were alive and in care, $4.7 \%$ died, $9.4 \%$ were lost to follow-up and $6.9 \%$ transferred.

Of the 19,645 patients, 17,272 (88\%) achieved virologic suppression on first-line ART and 1348 (9.9\%, 4.5/100 person-years) met the common failure definition (threshold $\geq 1000$ ). The median time from ART initiation until treatment failure among those who failed was 16 months (IQR:12-23), while the median time between the first and second detectable viral loads was 2.7 months (IQR:1.6-4.7).

\section{First-line Failure by Viral Load Threshold}

Figure 1 shows cumulative probabilities of virologic treatment failure, using different thresholds for confirmation in a Kaplan-Meier survival analysis. Between 6 months and five years on ART, using our most sensitive confirmatory threshold ( $\geq 400$ copies $/ \mathrm{ml}$ ), $16.9 \%$ (95\%CI:15.4-18.6\%) of patients failed. The proportion failing was similar when increasing this threshold to 1000 copies $/ \mathrm{ml}$, but was substantially less using thresholds of 5000 (10.0\%; 95\%CI:8.8\%-11.2\%) or 10,000 copies/ml (7.8\%; 95\% CI:6.6\%- 9.3\%, or 2.2 times less likely to meet the definition compared to a threshold of 400 copies $/ \mathrm{ml}$ ). This last estimate should be interpreted with caution, because no cohort used a threshold of 10,000 copies $/ \mathrm{ml}$ and so some patients failing at lower thresholds will have switched before reaching a threshold of 10,000 . When we limited the analysis for each threshold to only those cohorts which in practice defined failure as greater than or equal to the threshold examined and exclude the 10,000 group, results changed little (failure was 1.5 times greater comparing a threshold of 400 to a threshold of 5000). In sensitivity analyses including deaths following a single elevated viral load as failures, cumulative failure probability at 5 years on ART ranged from $16.7-24.7 \%$ depending on the definition used.

\section{Predictors of First-line Treatment Failure}

Table 3 shows associations of patient characteristics with treatment failure, using the common definition. In multivariable models (Table 3), age (per 10 year increase aHR:0.73; 95\%CI:0.68-0.79), NVP use (aHR:1.52; 95\%CI:1.34-1.73) and treatment provider were associated with failure. The association between NVP use and failure remained when 
modeling this separately for men and women (aHR:1.70, 95\%CI:1.30-2.21; $1.33,1.13-$ 1.56). One cohort had a substantially lower failure rate compared to the others. A CD4 count $<25$ at ART initiation cells/ $\mu 1$ was associated with a $60 \%$ increased risk of failure compared with those 100-199 cells/ $\mu$ l (aHR:1.60; 95\%CI:1.37-1.87). While gaps in treatment of 7 days or more were uncommon ( $\mathrm{N}=912,4.6 \%$ of patients), two or more gaps was associated with a 7-fold increased risk of failure (aHR:7.25; 95\%CI:4.95-10.6) and one gap associated with a 2-fold increased risk (aHR:2.46; 95\%CI:2.11-2.87). Results were very similar when using multiple imputation for missing data and when limited to patients who initially achieved viral suppression. Results were also similar for the outcome of failure or death except for an attenuation in the association with nevirapine in the initial regimen, the increased risk associated with 2 or more gaps in care was reduced (HR 2.74; 95\%CI:2.193.45 ) and a stronger association between later year of ART initiation and reduced failure/ death was observed (data not shown).

\section{Switching to Second-line}

Among the 1348 patients meeting the common failure definition, 62\% (833/1348) switched to second-line ART. Overall $10.1 \%$ of the cohort were switched $(9.0 \%-11.4 \%)$ to secondline between six months and five years on ART (Figure 1). Of those who completed at least six additional months of follow-up, 664 (74\%) switched at some point after failure. Those who switched did so a median of 4.6 months after failure (IQR:2.1-8.7). The majority (81\%) were switched to the government recommended regimen of AZT-ddi-LPVr.

Table 4 displays associations of patient characteristics with switching among those who failed according to their site's failure definition. In a multivariable analysis, CD4 count at failure was weakly associated with switching. Those with a higher rate of decline in CD4 count since failure were however more likely to switch. A CD4 \% drop of $-100 \%$ to $-51 \%$ vs. remaining relatively stable (-25\% to $25 \%$ ) was associated with a two-fold increased switch rate (aHR: $1.82 ; 95 \%$ CI:1.24-2.68) while a CD4 \% drop of $-50 \%$ to $0 \%$ compared to remaining stable ( $-25 \%$ to $25 \%$ ) was associated with a 1.3 -fold increased switch rate (95\%CI:0.93-1.77). There remained a two-fold difference between the cohorts with the fastest and slowest times to switching in virologically failing patients.

\section{DISCUSSION}

In one of the largest studies to date from the South African national treatment programme exploring the extent of virologic treatment failure, we found $8-17 \%$ of patients failed firstline therapy by five years on treatment using survival analysis depending on the definition of confirmed failure used, in line with findings from individual cohorts. ${ }^{6,31}$ There were expected delays both between a first elevated viral load and confirmation of treatment failure, and subsequent switching of therapy in those who switched (median of 2.7 and 4.6 months respectively). While nearly three-quarters of patients with confirmed virologic failure and at least six months of additional follow-up had switched, there was up to a twofold difference in time to switching between cohorts, with switching occurring faster in patients with rapidly falling CD4 counts.

\section{Definition of virologic failure}

South African and WHO guidelines suggest a pragmatic approach to defining virologic treatment failure which relies on confirmation of viraemia after attempts to optimize adherence. The World Health Organization 2010 guideline revisions recommend that when viral load monitoring is available, "A persistent viral load of $>5000$ copies $/ \mathrm{ml}$ confirms treatment failure." South African guidelines in operation at the time of this study used the same confirmatory threshold, but allowed for the first elevation to be above $400 \mathrm{copies} / \mathrm{ml}$. 
The approach has been shown to successfully select out patients with high levels of resistance warranting switching to second-line therapy, but low levels of cross-resistance between first and second-line regimens. ${ }^{32}$ As evidenced by this study however, the exact interpretation of this approach is varied and can profoundly impact the number of patients who meet the failure definition and require second-line therapy.

Three subsequent guideline changes in South Africa may impact the interpretation of these findings. ${ }^{28}$ In April 2010 the initial recommended NRTI backbone was changed from stavudine and lamivudine to tenofovir and lamivudine, while routine viral load testing frequency was dropped from six-monthly to annually beyond the first year on ART. The threshold for confirming virologic failure was lowered from 5,000 to 1,000 copies/ml with the confirmatory test now required within three months of the initial elevation.

Whereas the less frequent monitoring may increase delays to identifying patients failing virologically, with the introduction of tenofovir there may also now be fewer concerns about the accumulation of thymidine analogue mutations while viraemic, with the associated potential to compromise second-line therapy.

\section{Switching of failing patients}

A previous analysis from the IeDEA collaboration demonstrated switching occurred more frequently and at higher CD4 counts in sites with viral load monitoring. ${ }^{33}$ Mortality was lower and CD4 trajectories steeper in viral load sites, ${ }^{12,34}$ though this has not been consistent across studies. ${ }^{35}$ The current study provides further insight into the period between virologic failure confirmation and switching. A high proportion of patients who should be switched are switched, and compliance with guideline advice is more complete than is the case for children in South Africa. ${ }^{36}$ Nevertheless, the median delay of nearly 5 months between confirmation of failure and switch combined with inter-cohort differences, suggests that administrative and clinical factors are additionally impacting on compliance with switching guidelines. The strong association between CD4 count trajectory and switching further suggests that clinical judgment is a contributor to this variability.

Current data suggest PI-based second-line therapy is not being compromised by delays in switching resulting from South Africa's pragmatic virologic failure guidelines, with the majority of patients failing second-line therapy remaining susceptible to boosted lopinavir. ${ }^{37,38}$ The finding that most early failures on second-line are adherence-related supports the provision for a period of active adherence optimization prior to switching. Delays in switching on the other hand place patients at increased risk of illness and death through longer durations spent viraemic and at lower CD4 counts. ${ }^{12,13}$ An important followon analysis will therefore be to estimate the causal effect of delays in switching patients with confirmed virologic failing. ${ }^{39}$

\section{Associations with failure}

The associations with virologic failure we found mirror those found in individual cohorts. Measures of advanced disease (CD4 count, WHO stage, viral load) were associated with failure. We found a strong association with treatment interruptions, perhaps serving both as a proxy for poor adherence and as a consequence of the long half-life of NNRTIs which remain in circulation longer than the other drugs following unplanned interruptions. ${ }^{40,41} \mathrm{We}$ again found NVP as choice of NNRTI was associated with virologic failure, a consistent finding across observational studies from different settings, ${ }^{31,42-44}$ and is not in conflict with the clinical trial data for African sites. ${ }^{45,46}$ 


\section{Limitations}

Our study has several limitations. First we lacked good PMTCT data to be able to examine the role of single-dose NVP exposure in treatment failure. Second, confounding by indication could have occurred, particularly in the relationship between nevirapine use and failure in sites where efavirenz was more commonly used. Next, although we observed modest differences between cohorts in the completion and frequency of viral load testing, failure to test or report viral load results according to guidelines would reduce the probability of meeting failure definitions. We also found the overall failure rate was sensitive to assumptions made about whether patients with only a single detectable viral load before death were truly failures. Also differences in how failure was defined by each cohort could have led to underestimates of failure rates using definitions with higher thresholds if patients with detectable viral loads below the threshold were switched before they could reach a higher threshold. Differences in follow-up time between cohorts may also explain some of the differences in failure rates observed. Finally, we had no data on two important potential predictors of treatment failure, adherence ${ }^{47}$ and prior resistance.

In conclusion, future treatment guidelines revisions should make explicit the rationale for the thresholds chosen to define and confirm virologic failure in light of our finding that these profoundly on the proportion of patients who meet failure definitions, and resultant costs of second-line treatment. Although guidance on switching failing patients is generally followed, there remains considerable variability in time to switching after failure, due to both clinician and administrative factors. Future studies should investigate the impact failure definitions and delays in switching have on subsequent treatment outcomes.

\section{Acknowledgments}

\section{Source of Funding:}

The project described was supported by Grant Number U01AI069924 from NIH (NIAID, NICHD, NCI, Principal Investigators Egger and Davies). Dr. Fox was funded by Award Number K01AI083097 from the National Institute of Allergy And Infectious Diseases. Dr. Hernán was funded by NIH R01 AI073127. Professor Sterne was supported by UK MRC grant G0700820. Dr Keiser was funded by a PROSPER fellowship by the Swiss National Science Foundation (Grant 32333B_131629). The findings are solely the responsibility of the authors and do not necessarily represent the official views of the NIH. The funders had no role in study design, data collection and analysis, decision to publish, or preparation of the manuscript. We thank all the patients whose data was used in this analysis. We also thank all staff at participating sites for preparation of data contributed to the IeDEA Southern Africa collaboration. Many thanks to Nicola Maxwell for preparing the combined data for analysis, to Morna Cornell and Claire Graber for project management and to Michael Schomaker for advice and technical assistance with the analysis. Matthew Fox had full access to all the data in the study and takes responsibility for the integrity of the data and the accuracy of the data analysis.

\section{References}

1. World Health Organization. Antiretroviral therapy for HIV infection in adults and adolescents Recommendations for a public health approach, 2010 revision. Geneva: 2010.

2. Boulle A, Van Cutsem G, Hilderbrand K, et al. Seven-year experience of a primary care antiretroviral treatment programme in Khayelitsha, South Africa. AIDS. Feb 20; 2010 24(4):563572. [PubMed: 20057311]

3. Stringer JS, Zulu I, Levy J, et al. Rapid scale-up of antiretroviral therapy at primary care sites in Zambia: feasibility and early outcomes. JAMA. Aug 16; 2006 296(7):782-793. [PubMed: 16905784]

4. Sanne IM, Westreich D, Macphail AP, Rubel D, Majuba P, Van Rie A. Long term outcomes of antiretroviral therapy in a large HIV/AIDS care clinic in urban South Africa: a prospective cohort study. J Int AIDS Soc. 2009; 12:38. [PubMed: 20017918] 
5. Ferradini L, Jeannin A, Pinoges L, et al. Scaling up of highly active antiretroviral therapy in a rural district of Malawi: an effectiveness assessment. Lancet. Apr 22; 2006 367(9519):1335-1342. [PubMed: 16631912]

6. Nglazi MD, Lawn SD, Kaplan R, et al. Changes in programmatic outcomes during 7 years of scaleup at a community-based antiretroviral treatment service in South Africa. J Acquir Immune Defic Syndr. Jan 1; 2011 56(1):e1-8. [PubMed: 21084996]

7. May M, Boulle A, Phiri S, et al. Prognosis of patients with HIV-1 infection starting antiretroviral therapy in sub-Saharan Africa: a collaborative analysis of scale-up programmes. Lancet. Aug 7; 2010 376(9739):449-457. [PubMed: 20638120]

8. Fox MP, Ive P, Long L, Maskew M, Sanne I. High rates of survival, immune reconstitution, and virologic suppression on second-line antiretroviral therapy in South Africa. J Acquir Immune Defic Syndr. Apr 1; 2010 53(4):500-506. [PubMed: 19838128]

9. Pujades-Rodriguez M, O’Brien D, Humblet P, Calmy A. Second-line antiretroviral therapy in resource-limited settings: the experience of Medecins Sans Frontieres. AIDS. Jul 11; 2008 22(11): 1305-1312. [PubMed: 18580610]

10. Palombi L, Marazzi MC, Guidotti G, et al. Incidence and predictors of death, retention, and switch to second-line regimens in antiretroviral- treated patients in sub-Saharan African Sites with comprehensive monitoring availability. Clin Infect Dis. Jan 1; 2009 48(1):115-122. [PubMed: 20380075]

11. Renaud-Thery F, Nguimfack BD, Vitoria M, et al. Use of antiretroviral therapy in resource-limited countries in 2006: distribution and uptake of first- and second-line regimens. AIDS. Jul; 2007 21( Suppl 4):S89-95. [PubMed: 17620758]

12. Keiser O, Chi B, Gsponer T, et al. Outcomes of antiretroviral treatment in programmes with and without routine viral load monitoring in Southern Africa. AIDS. 2011 epub ahead of print.

13. Keiser O, Tweya H, Braitstein $\mathrm{P}$, et al. Mortality after failure of antiretroviral therapy in subSaharan Africa. Trop Med Int Health. Feb; 2010 15(2):251-258. [PubMed: 20003034]

14. WHO U, UNICEF. Towards universal access: scaling up priority HIV/AIDS interventions in the health sector-progress report. Sep. 2009

15. Boyd MA. Current and future management of treatment failure in low- and middle-income countries. Curr Opin HIV AIDS. Jan; 2010 5(1):83-89. [PubMed: 20046152]

16. Harries AD, Zachariah R, van Oosterhout JJ, et al. Diagnosis and management of antiretroviraltherapy failure in resource-limited settings in sub-Saharan Africa: challenges and perspectives. Lancet Infect Dis. Jan; 2010 10(1):60-65. [PubMed: 20129150]

17. Keiser O, MacPhail P, Boulle A, et al. Accuracy of WHO CD4 cell count criteria for virological failure of antiretroviral therapy. Trop Med Int Health. Oct; 2009 14(10):1220-1225. [PubMed: 19624478]

18. van Oosterhout JJ, Brown L, Weigel R, et al. Diagnosis of antiretroviral therapy failure in Malawi: poor performance of clinical and immunological WHO criteria. Trop Med Int Health. Aug; 2009 14(8):856-861. [PubMed: 19552661]

19. Moore DM, Awor A, Downing R, et al. CD4+ T-cell count monitoring does not accurately identify HIV-infected adults with virologic failure receiving antiretroviral therapy. J Acquir Immune Defic Syndr. Dec 15; 2008 49(5):477-484. [PubMed: 18989232]

20. Mee P, Fielding KL, Charalambous S, Churchyard GJ, Grant AD. Evaluation of the WHO criteria for antiretroviral treatment failure among adults in South Africa. AIDS. Oct 1; 2008 22(15):19711977. [PubMed: 18784460]

21. Bisson GP, Gross R, Strom JB, et al. Diagnostic accuracy of CD4 cell count increase for virologic response after initiating highly active antiretroviral therapy. AIDS. Aug 1; 2006 20(12):16131619. [PubMed: 16868442]

22. Reynolds SJ, Nakigozi G, Newell K, et al. Failure of immunologic criteria to appropriately identify antiretroviral treatment failure in Uganda. AIDS. Mar 27; 2009 23(6):697-700. [PubMed: 19209067]

23. Egger M, Ekouevi DK, Williams C, et al. Cohort Profile: The international epidemiological databases to evaluate AIDS (IeDEA) in sub-Saharan Africa. Int J Epidemiol. May 18.2011 
24. Cornell M, Grimsrud A, Fairall L, et al. Temporal changes in programme outcomes among adult patients initiating antiretroviral therapy across South Africa, 2002-2007. AIDS. 2010; 24(14): 2263-2270. [PubMed: 20683318]

25. National Department of Health, Republic of South Africa. South African National Antiretroviral Treatment Guidelines. First edition. Pretoria: National Department of Health; 2004.

26. National Department of Health, Republic of South Africa. Clinical Guidelines for the Management of HIV\&AIDS in Adults and Adolescents. Pretoria: National Department of Health; 2010.

27. Cornell M, Technau K, Fairall L, et al. Monitoring the South African National Antiretroviral Treatment Programme, 2003-2007: The IeDEA Southern Africa collaboration. SAMJ S Afr Med J. Sep; 2009 99(9):653-660.

28. National Department of Health RoSA. Clinical Guidelines for the Management of HIV\&AIDS in Adults and Adolescents. 2010.

29. van Buuren S, Boshuizen HC, Knook DL. Multiple imputation of missing blood pressure covariates in survival analysis. Stat Med. Mar 30; 1999 18(6):681-694. [PubMed: 10204197]

30. Rubin DB. Multiple imputation after 18+ years. J Am Stat Assoc. Jun; 1996 91(434):473-489.

31. Boulle A, van Cutsem G, Hilderbrand K, et al. Seven year experience of a primary care antiretroviral treatment programme in Khayelitsha, South Africa. AIDS. 2010; 24(14):563-572. [PubMed: 20057311]

32. Orrell C, Walensky RP, Losina E, Pitt J, Freedberg KA, Wood R. HIV type-1 clade C resistance genotypes in treatment-naive patients and after first virological failure in a large community antiretroviral therapy programme. Antivir Ther. 2009; 14(4):523-531. [PubMed: 19578237]

33. Keiser O, Tweya H, Boulle A, et al. Switching to second-line antiretroviral therapy in resourcelimited settings: comparison of programmes with and without viral load monitoring. AIDS. Jun 15; 2009 23(14):1867-1874. [PubMed: 19531928]

34. Keiser O, Chi BH, Gsponer T, et al. Outcomes of antiretroviral treatment in programmes with and without routine viral load monitoring in Southern Africa. Aids. Jun 15.2011

35. Mermin J, Ekwaru JP, Were W, et al. Utility of routine viral load, CD4 cell count, and clinical monitoring among adults with HIV receiving antiretroviral therapy in Uganda: randomised trial. BMJ. 2011; 343:d6792. [PubMed: 22074711]

36. Davies MA, Moultrie H, Eley B, et al. Virologic failure and second-line antiretroviral therapy in children in South Africa--the IeDEA Southern Africa collaboration. J Acquir Immune Defic Syndr. Mar 1; 2011 56(3):270-278. [PubMed: 21107266]

37. Hosseinipour MC, Kumwenda JJ, Weigel R, et al. Second-line treatment in the Malawi antiretroviral programme: high early mortality, but good outcomes in survivors, despite extensive drug resistance at baseline. HIV Med. Sep; 2010 11(8):510-518. [PubMed: 20345885]

38. Wallis CL, Mellors JW, Venter WD, Sanne I, Stevens W. Protease Inhibitor Resistance Is ncommon in HIV-1 Subtype C Infected Patients on Failing Second-Line Lopinavir/r-Containing Antiretroviral Therapy in South Africa. AIDS Res Treat. 2011; 2011:769627. [PubMed: 21490784]

39. Hernan MA, Lanoy E, Costagliola D, Robins JM. Comparison of dynamic treatment regimes via inverse probability weighting. Basic Clin Pharmacol Toxicol. Mar; 2006 98(3):237-242. [PubMed: 16611197]

40. Fox Z, Phillips A, Cohen C, et al. Viral resuppression and detection of drug resistance following interruption of a suppressive non-nucleoside reverse transcriptase inhibitor-based regimen. AIDS. Nov 12; 2008 22(17):2279-2289. [PubMed: 18981767]

41. Bansi LK, Benzie AA, Phillips AN, et al. Are previous treatment interruptions associated with higher viral rebound rates in patients with viral suppression? AIDS. Jan 30; 2008 22(3):349-356. [PubMed: 18195561]

42. Nachega JB, Hislop M, Dowdy DW, Chaisson RE, Regensberg L, Maartens G. Adherence to nonnucleoside reverse transcriptase inhibitor-based HIV therapy and virologic outcomes. Ann Intern Med. Apr 17; 2007 146(8):564-573. [PubMed: 17438315]

43. Keiser P, Nassar N, White C, Koen G, Moreno S. Comparison of nevirapine- and efavirenzcontaining antiretroviral regimens in antiretroviral-naive patients: a cohort study. HIV Clin Trials. Jul-Aug;2002 3(4):296-303. [PubMed: 12187503] 
44. Cozzi-Lepri A, Phillips AN, d'Arminio Monforte A, et al. Virologic and immunologic response to regimens containing nevirapine or efavirenz in combination with 2 nucleoside analogues in the Italian Cohort Naive Antiretrovirals (I.Co.N.A.) study. J Infect Dis. Apr 15; 2002 185(8):10621069. [PubMed: 11930316]

45. van Leth F, Phanuphak P, Ruxrungtham K, et al. Comparison of first-line antiretroviral therapy with regimens including nevirapine, efavirenz, or both drugs, plus stavudine and lamivudine: a randomised open-label trial, the 2NN Study. Lancet. Apr 17; 2004 363(9417):1253-1263. [PubMed: 15094269]

46. Wester CW, Thomas AM, Bussmann H, et al. Non-nucleoside reverse transcriptase inhibitor outcomes among combination antiretroviral therapy-treated adults in Botswana. Aids. Jan; 2010 24( Suppl 1):S27-36. [PubMed: 20023437]

47. Ramadhani HO, Thielman NM, Landman KZ, et al. Predictors of incomplete adherence, virologic failure, and antiviral drug resistance among HIV-infected adults receiving antiretroviral therapy in Tanzania. Clin Infect Dis. Dec 1; 2007 45(11):1492-1498. [PubMed: 17990233] 


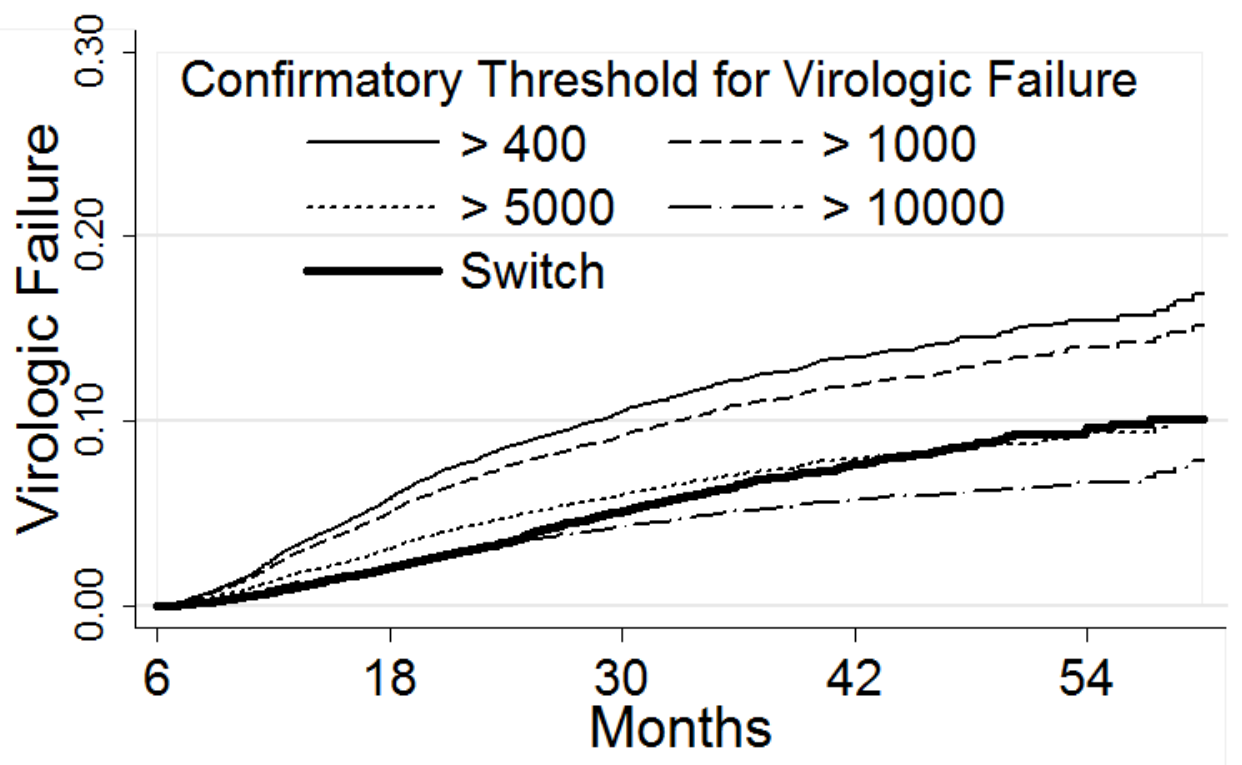

$\mathrm{N}$ - (Event)

\begin{tabular}{|c|c|c|c|c|c|c|c|c|c|}
\hline$>400$ & 19611 & (890) & 11704 & (458) & 5921 & (140) & 2447 & (32) & 472 \\
\hline 1000 & 19611 & (775) & 11804 & (405) & 6012 & (131) & 2495 & (33) & 482 \\
\hline 500 & 19611 & (477) & 12058 & (282) & 6230 & (93) & 2598 & (20) & 505 \\
\hline 1000 & 19611 & (337) & 12170 & (204) & 6356 & (67) & 2672 & (14) & 527 \\
\hline Swit & & (315) & 8172 & ) & & (121) & 2602 & (31) & 507 \\
\hline
\end{tabular}

Figure 1. 


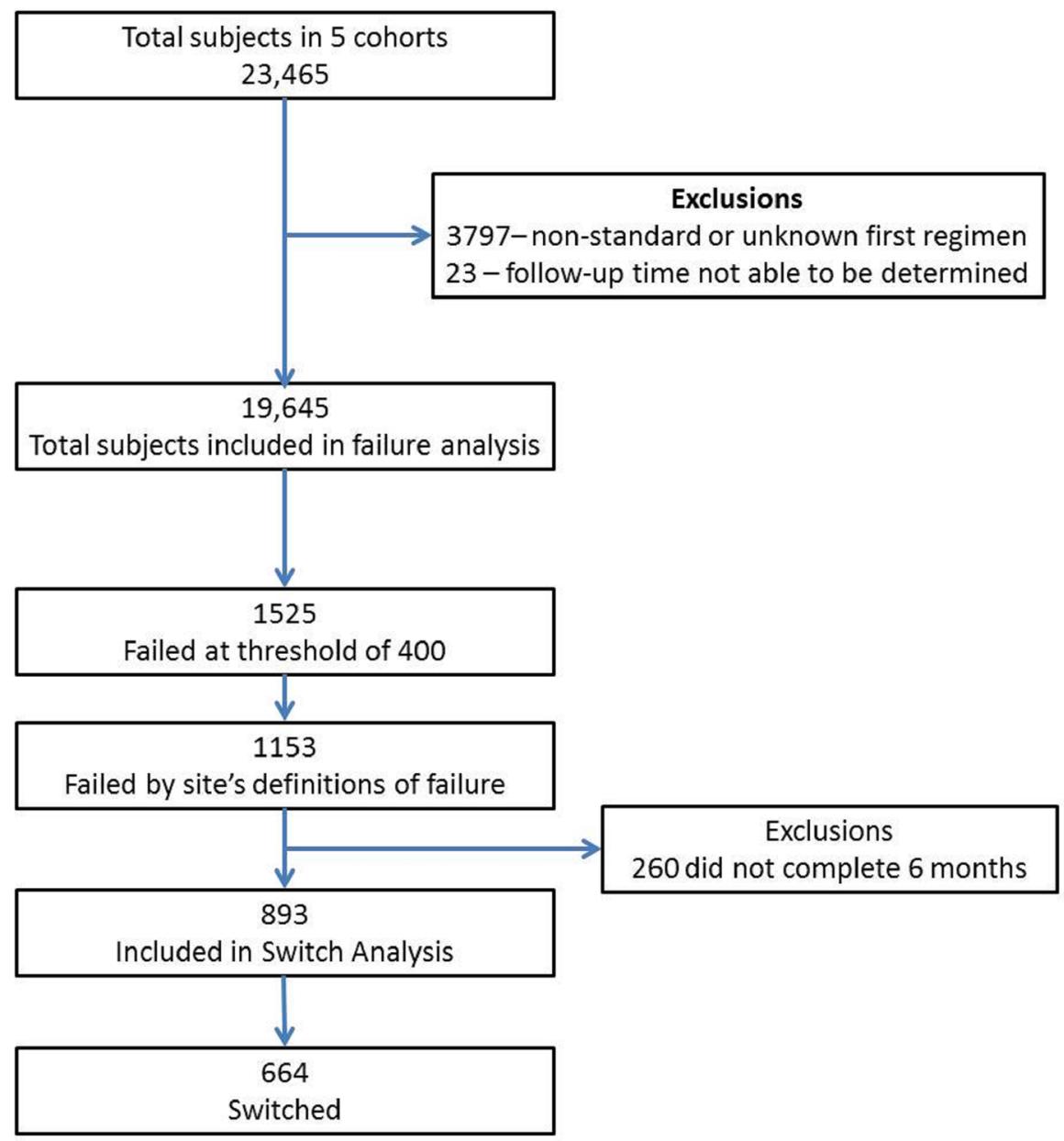

Appendix 1.

Study Profile of Virologic Treatment Failure and Switching to Second-line Antiretroviral Therapy in the IeDEA-SA South Africa Cohort 

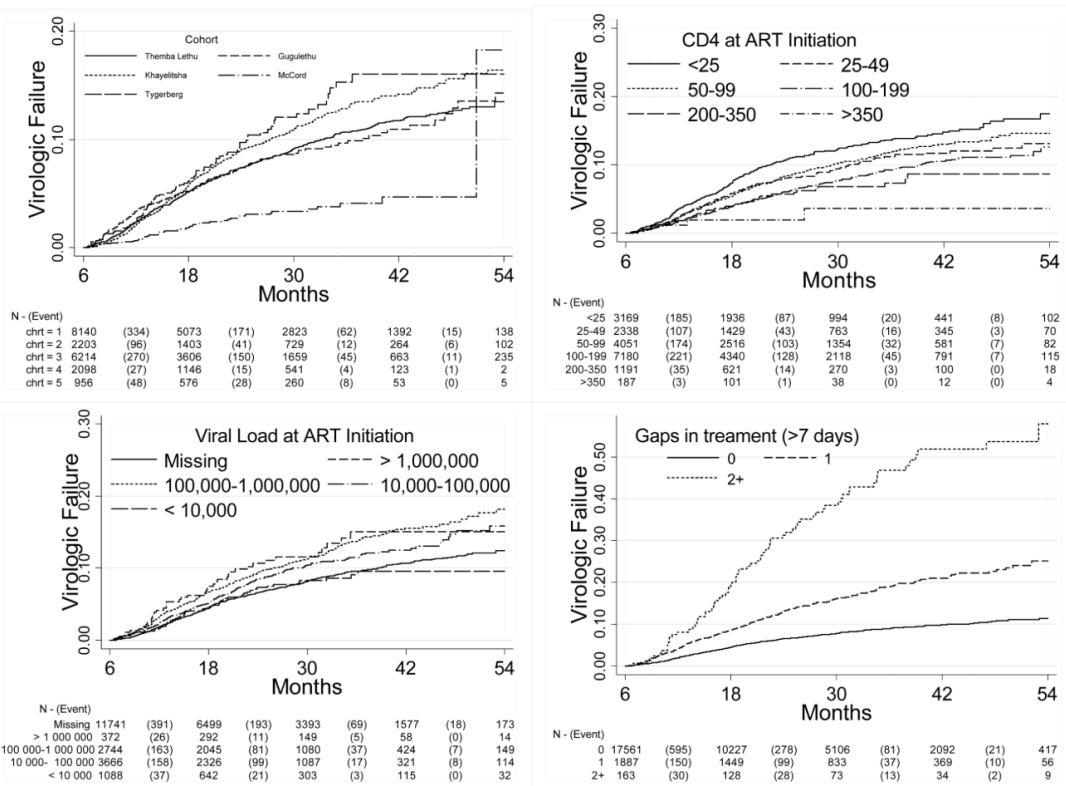

\section{Appendix 2.}

Kaplan-Meier Curves of predictors of failure stratified by a) cohort, b) CD4 count at ART initiation, c) viral load at ART initiation, and d) gaps in treatment

Log-rank $\mathrm{p}$-value for cohort $(\mathrm{p}<0.0001), \mathrm{CD} 4$ count at ART initiation $(\mathrm{p}<0.0001)$, viral load at ART initiation $(\mathrm{p}=0.0011)$, and gaps in treatment $(\mathrm{p}<0.0001)$ 


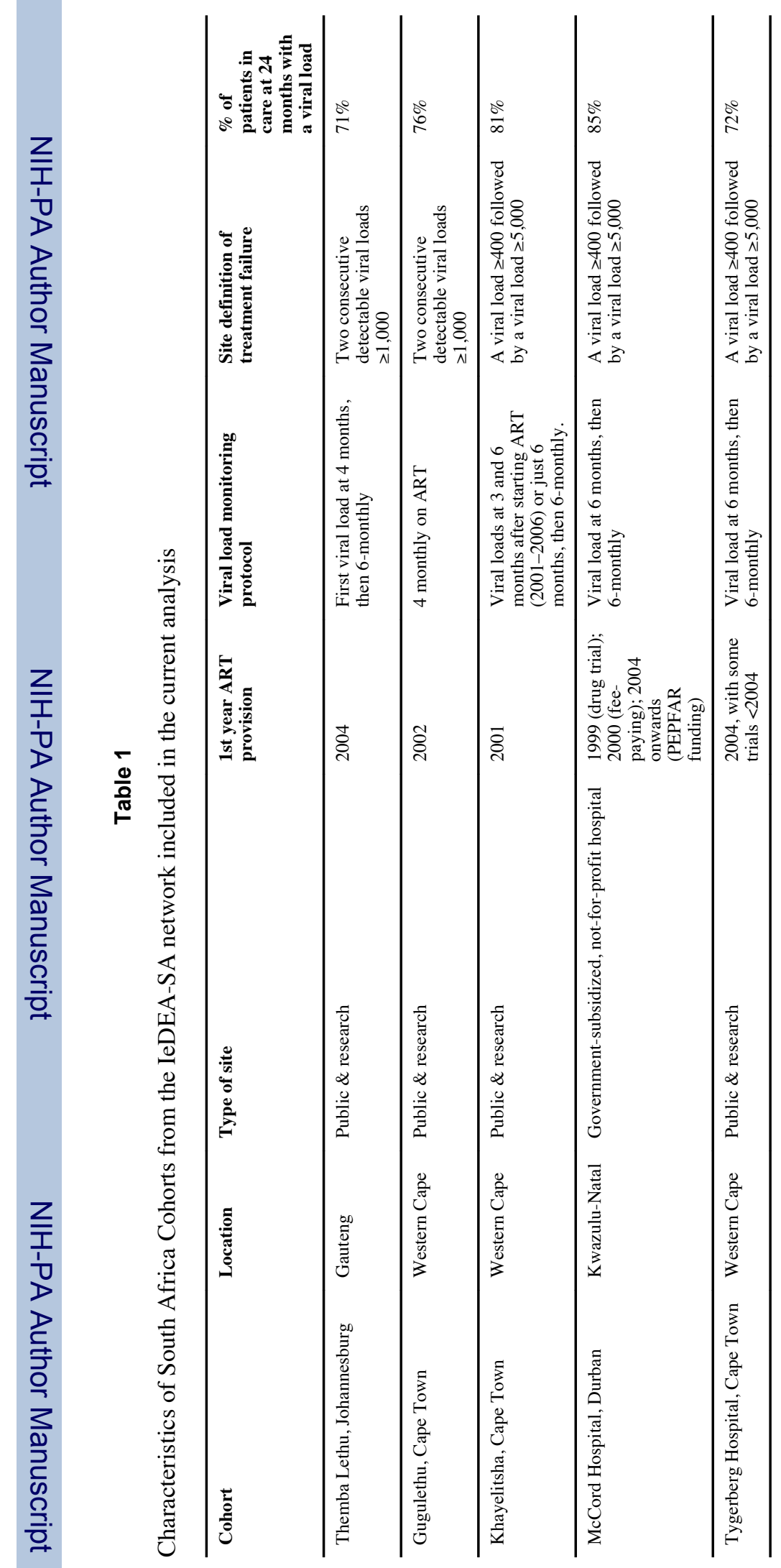


Table 2

Characteristics of eligible patients participating in South African Cohorts in the IeDEA-SA network

\begin{tabular}{|c|c|c|}
\hline Patient characteristic & Exposure & Full cohort ${ }^{¥}$ \\
\hline \multirow[t]{5}{*}{ Treatment provider } & Themba Lethu & $8157(41.5 \%)$ \\
\hline & Gugulethu & $2210(11.2 \%)$ \\
\hline & Khayelitsha & $6220(31.7 \%)$ \\
\hline & McCord & $2102(10.7 \%)$ \\
\hline & Tygerberg & $956(4.9 \%)$ \\
\hline \multicolumn{3}{|l|}{ Gender } \\
\hline & Male & $6665(33.9 \%)$ \\
\hline & Female & $12980(66.1 \%)$ \\
\hline \multicolumn{3}{|l|}{ Age at ART initiation } \\
\hline & $<20$ & $137(0.7 \%)$ \\
\hline & $20-29$ & $5190(26.4 \%)$ \\
\hline & $30-39$ & $9046(46.0 \%)$ \\
\hline & $40-49$ & $4005(20.4 \%)$ \\
\hline & $50+$ & $1267(6.4 \%)$ \\
\hline \multicolumn{3}{|l|}{ Year of ART initiation } \\
\hline & $<2004$ & $925(4.7 \%)$ \\
\hline & 2004 & $3345(17.0 \%)$ \\
\hline & 2005 & $5137(26.1 \%)$ \\
\hline & 2006 & $5743(29.2 \%)$ \\
\hline & 2007 & $3812(19.4 \%)$ \\
\hline & $2008+$ & $683(3.5 \%)$ \\
\hline \multicolumn{3}{|l|}{ First ART } \\
\hline & D4T-3TC-EFV & $13425(68.3 \%)$ \\
\hline & AZT-3TC-EFV & $977(5.0 \%)$ \\
\hline & D4T-3TC-NVP & $4190(21.3 \%)$ \\
\hline & AZT-3TC-NVP & $1053(5.4 \%)$ \\
\hline \multicolumn{3}{|c|}{ WHO stage at ART initiation } \\
\hline & Stage I & $5296(30.6 \%)$ \\
\hline & Stage II & $1230(7.1 \%)$ \\
\hline & Stage III & $7261(42.0 \%)$ \\
\hline & Stage IV & $3521(20.3 \%)$ \\
\hline & Missing & 2337 \\
\hline \multicolumn{3}{|c|}{ Patient on TB treatment at start of HAART? } \\
\hline & No & $8651(63.9 \%)$ \\
\hline & Yes & $4893(36.1 \%)$ \\
\hline & Missing & 6101 \\
\hline
\end{tabular}




\begin{tabular}{|c|c|c|}
\hline Patient characteristic & Exposure & Full cohort ${ }^{¥}$ \\
\hline \multicolumn{3}{|l|}{ Exposed to PMTCT } \\
\hline & No & $3745(34.3 \%)$ \\
\hline & Yes & $499(4.6 \%)$ \\
\hline & $\mathrm{N} / \mathrm{A}$ & $6665(61.1 \%)$ \\
\hline & Missing & 8736 \\
\hline \multicolumn{3}{|l|}{ Pregnant at start of HAART? } \\
\hline & Yes & $510(3.9 \%)$ \\
\hline & No & $5798(44.7 \%)$ \\
\hline & NA & $6665(51.4 \%)$ \\
\hline & Missing & 7072 \\
\hline \multicolumn{3}{|l|}{ CD4 count at ART initiation (cells $/ \mathrm{mm}^{3}$ ) } \\
\hline & $<25$ & $3172(17.5 \%)$ \\
\hline & $25-49$ & $2341(12.9 \%)$ \\
\hline & $50-99$ & $4061(22.4 \%)$ \\
\hline & 100-199 & $7193(39.6 \%)$ \\
\hline & $200-349$ & $1194(6.6 \%)$ \\
\hline & $>350$ & $187(1.0 \%)$ \\
\hline & Missing & 1497 \\
\hline Median (IQR)CD4 at ART initiation & & $93(39-155)$ \\
\hline \multicolumn{3}{|l|}{ Viral load at ART initiation } \\
\hline & $>1,000,000$ & $372(4.7 \%)$ \\
\hline & $100,000-1,000,000$ & $2748(34.9 \%)$ \\
\hline & $10,000-100,000$ & $3672(46.6 \%)$ \\
\hline & $<10,000$ & $1093(13.9 \%)$ \\
\hline & Missing & 11760 \\
\hline \multirow[t]{4}{*}{ Viral load at ART initiation $\times 1000$ (Median $(\mathrm{IQR}))$ Drug interruptions of $7+$ days } & & $65(20-220)$ \\
\hline & 0 & $18732(95.4 \%)$ \\
\hline & 1 & $885(4.5 \%)$ \\
\hline & $2+$ & $28(0.1 \%)$ \\
\hline \multicolumn{3}{|l|}{ Outcome } \\
\hline & Alive & $15362(79.0 \%)$ \\
\hline & Died & $921(4.7 \%)$ \\
\hline & LTFU & $1834(9.4 \%)$ \\
\hline & Transferred & $1339(6.9 \%)$ \\
\hline Median (IQR) weight at ART initiation (kgs) [number of measurements] & & $59(52-67)[14120]$ \\
\hline
\end{tabular}


Table 3

Predictors of first-line antiretroviral virologic treatment failure in the IeDEA-SA cohorts in South Africa

\begin{tabular}{lllll}
\hline Group & Rate/100py (N/p-years) & Crude Model & Adjusted Model & Adjusted Model with Multiple Imputation \\
\hline Treatment Provider & & $\mathrm{N}=19645$ & $\mathrm{~N}=19645$ & \\
\hline Themba Lethu & $3.46(583 / 16871)$ & Reference & Reference & Reference \\
Gugulethu & $3.35(155 / 4628)$ & $0.96(0.80-1.15)$ & $0.73(0.56-0.95)$ & $0.73(0.57-0.93)$ \\
Khayelitsha & $3.90(479 / 12269)$ & $1.15(1.02-1.30)$ & $0.82(0.67-1.00)$ & $0.83(0.63-1.10)$ \\
McCord & $1.24(47 / 3798)$ & $0.37(0.27-0.50)$ & $0.29(0.16-0.53)$ & $0.25(0.17-0.37)$ \\
Tygerberg & $4.58(84 / 1834)$ & $1.31(1.04-1.65)$ & $0.86(0.62-1.20)$ & $0.85(0.66-1.10)$ \\
Age & & & & \\
\hline 10 year increase & N/A & $0.73(0.68-0.78)$ & $0.73(0.68-0.79)$ & $0.73(0.68-0.79)$ \\
Gender & & & & \\
\hline
\end{tabular}

\begin{tabular}{lllll}
\hline Male & $3.34(434 / 12986)$ & Reference & Reference & Reference \\
Female & $3.46(914 / 26414)$ & $1.02(0.91-1.14)$ & $0.95(0.81-1.12)$ & $0.86(0.76-0.97)$
\end{tabular}

Year of initiation of 1st line ART

\begin{tabular}{lllll}
\hline$<2004$ & $3.92(148 / 3776)$ & Reference & Reference & Reference \\
2004 & $3.54(357 / 10084)$ & $0.82(0.67-1.00)$ & $0.88(0.72-1.08)$ & $0.88(0.72-1.08)$ \\
2005 & $3.84(459 / 11967)$ & $0.86(0.71-1.04)$ & $0.97(0.79-1.19)$ & $0.99(0.81-1.21)$ \\
2006 & $3.43(316 / 9203)$ & $0.86(0.70-1.06)$ & $0.96(0.77-1.20)$ & $0.98(0.78-1.22)$ \\
$2007+$ & $1.56(68 / 4370)$ & $0.64(0.47-0.86)$ & $0.72(0.52-1.00)$ & $0.78(0.56-1.07)$ \\
NNRTI in first-line ART regimen & & &
\end{tabular}

NNRTI in first-line ART regimen

\begin{tabular}{lllll}
\hline EFV & $3.07(897 / 29177)$ & Reference & Reference & Reference \\
NVP & $4.41(451 / 10224)$ & $1.46(1.30-1.64)$ & $1.45(1.26-1.66)$ & $1.48(1.29-1.71)$
\end{tabular}

TB treatment at ART Start

\begin{tabular}{|c|c|c|c|c|}
\hline Yes & $2.85(258 / 9068)$ & $0.83(0.72-0.96)$ & $1.11(0.93-1.32)$ & $1.11(0.93-1.32)$ \\
\hline \multicolumn{5}{|c|}{ CD4 count at ART initiation (cells $/ \mathrm{mm}^{3}$ ) } \\
\hline $25-49$ & $3.51(169 / 4812)$ & $1.24(1.04-1.48)$ & $1.21(1.01-1.45)$ & $1.24(1.03-1.51)$ \\
\hline $50-99$ & $3.78(318 / 8410)$ & $1.32(1.14-1.53)$ & $1.29(1.11-1.50)$ & $1.31(1.13-1.51)$ \\
\hline $100-199$ & $2.84(402 / 14134)$ & Reference & Reference & Reference \\
\hline $200-349$ & $2.46(52 / 2113)$ & $0.90(0.67-1.20)$ & $0.91(0.68-1.22)$ & $0.89(0.67-1.19)$ \\
\hline \multicolumn{5}{|c|}{ Viral Load at ART initiation } \\
\hline$<10,000$ & $2.88(61 / 2120)$ & Reference & Reference & Reference \\
\hline $10,000-99,999$ & $3.81(283 / 7422)$ & $1.29(0.98-1.70)$ & $1.10(0.83-1.46)$ & $1.14(0.92-1.41)$ \\
\hline
\end{tabular}




\begin{tabular}{lllll}
\hline Group & Rate/100py (N/p-years) & Crude Model & Adjusted Model & Adjusted Model with Multiple Imputation \\
\hline $100,000-999,999$ & $4.48(289 / 6449)$ & $1.47(1.12-1.94)$ & $1.20(0.90-1.60)$ & $1.33(1.10-1.61)$ \\
$\geq 1,000,000$ & $4.73(42 / 888)$ & $1.53(1.03-2.27)$ & $1.19(0.79-1.79)$ & $1.40(1.01-1.95)$ \\
Missing & $2.99(673 / 22521)$ & $1.05(0.81-1.36)$ & $0.98(0.74-1.29)$ & N/A \\
WHO Stage at ART initiation & & & \\
\hline I/II & $2.96(384 / 12995)$ & Reference & Reference & Reference \\
III/IV & $4.04(896 / 22175)$ & $1.35(1.20-1.52)$ & $1.28(1.12-1.47)$ & $1.36(1.08-1.72)$ \\
Missing & $1.61(68 / 4231)$ & $0.55(0.42-0.71)$ & $1.40(0.84-2.32)$ & N/A \\
ART treatment interruptions of $>7$ days & & & \\
\hline 0 & $3.02(1000 / 37045)$ & Reference & Reference & Reference \\
1 & $9.02(202 / 2239)$ & $2.36(2.03-2.75)$ & $2.46(2.11-2.87)$ & $2.49(2.13-2.91)$ \\
$2+$ & $24.17(28 / 116)$ & $6.74(4.62-9.83)$ & $7.25(4.95-10.62)$ & $7.22(4.93-10.6)$ \\
\hline
\end{tabular}


Table 4

Predictors of Switch among those who Failed

\begin{tabular}{|c|c|c|}
\hline Predictor & Crude Model N=893 & Adjusted Model N=893 \\
\hline \multicolumn{3}{|l|}{ Treatment provider } \\
\hline Themba Lethu & Reference & Reference \\
\hline Gugulethu & $0.62(0.49-0.79)$ & $0.61(0.48-0.78)$ \\
\hline Khayelitsha & $0.54(0.45-0.65)$ & $0.51(0.40-0.65)$ \\
\hline McCord & $0.97(0.52-1.82)$ & $0.91(0.48-1.73)$ \\
\hline Tygerberg & $1.07(0.74-1.55)$ & $1.08(0.73-1.61)$ \\
\hline \multicolumn{3}{|l|}{ Age } \\
\hline 10 year increase & $1.03(0.94-1.13)$ & $0.96(0.87-1.06)$ \\
\hline \multicolumn{3}{|l|}{ Gender } \\
\hline Male & Reference & Reference \\
\hline Female & $0.94(0.80-1.10)$ & $0.96(0.81-1.14)$ \\
\hline \multicolumn{3}{|c|}{ Year failed first-line ART } \\
\hline$<2004$ & Reference & Reference \\
\hline 2004 & $0.91(0.53-1.56)$ & $0.93(0.54-1.61)$ \\
\hline 2005 & $0.99(0.66-1.48)$ & $0.77(0.51-1.17)$ \\
\hline 2006 & $0.93(0.64-1.36)$ & $0.61(0.40-0.93)$ \\
\hline $2007+$ & $1.50(1.03-2.19)$ & $0.97(0.63-1.49)$ \\
\hline \multicolumn{3}{|c|}{ Years on ART at failure } \\
\hline$<1$ & Reference & Reference \\
\hline $1-1.9$ & $0.97(0.82-1.15)$ & $0.99(0.83-1.19)$ \\
\hline $2-2$ & $1.23(0.96-1.57)$ & $1.09(0.84-1.41)$ \\
\hline $3-3.9$ & $0.92(0.61-1.39)$ & $0.91(0.59-1.40)$ \\
\hline $4+$ & $1.26(0.56-2.84)$ & $1.65(0.72-3.76)$ \\
\hline \multicolumn{3}{|c|}{ Current CD4 (cells $\left./ \mathrm{mm}^{3}\right)$} \\
\hline $350+$ & Reference & Reference \\
\hline $200-349$ & $1.19(0.94-1.51)$ & $1.02(0.79-1.31)$ \\
\hline $100-199$ & $1.27(0.99-1.62)$ & $1.04(0.80-1.35)$ \\
\hline $50-99$ & $1.47(1.09-1.99)$ & $1.18(0.85-1.64)$ \\
\hline $0-49$ & $1.57(1.14-2.16)$ & $1.01(0.70-1.46)$ \\
\hline \multicolumn{3}{|c|}{$\% \mathrm{CD} 4$ change from failure } \\
\hline$-100 \%$ to $-51 \%$ & $1.72(1.22-2.42)$ & $1.82(1.24-2.68)$ \\
\hline$-50 \%$ to $-26 \%$ & $1.11(0.81-1.52)$ & $1.28(0.93-1.77)$ \\
\hline$-25 \%$ to $25 \%$ & Reference & Reference \\
\hline$>25 \%$ & $0.54(0.41-0.70)$ & $0.50(0.38-0.66)$ \\
\hline Missing & $0.50(0.28-0.89)$ & $0.59(0.32-1.09)$ \\
\hline
\end{tabular}




\begin{tabular}{lll}
\hline Predictor & Crude Model N=893 & Adjusted Model N=893 \\
\hline$<10,000$ & Reference & Reference \\
$10,000-99,999$ & $1.12(0.96-1.31)$ & $1.15(0.97-1.36)$ \\
$100,000-999,999$ & $0.61(0.42-0.89)$ & $0.84(0.56-1.27)$ \\
$\geq 1,000,000$ & $3.14(0.78-12.64)$ & $1.92(0.45-8.21)$ \\
\hline
\end{tabular}

